\title{
OnehopMANET: One-hop Structured P2P over Mobile ad hoc Networks
}

\author{
Mohammad Al Mojamed \\ Computing Science and Mathematics \\ University of Stirling \\ Stirling, Scotland \\ mma@cs.stir.ac.uk
}

\author{
Mario Kolberg \\ Computing Science and Mathematics \\ University of Stirling \\ Stirling, Scotland \\ mko@cs.stir.ac.uk
}

\begin{abstract}
There are many common characteristics between P2P (Peer to Peer) overlay networks and MANET (mobile ad hoc networks). Previous work has shown that when used together, the two approaches complement each other and performance synergies can be exploited. While MANET provide wireless connectivity without depending on any pre-existing infrastructure, $P 2 P$ overlays provide data storage/retrieval functionality. On the other hand, both approaches face common challenges: maintaining connectivity in dynamic and decentralized networks. This paper proposes OnehopMANET as a structured P2P over MANET the uses cross-layering with a proactive underlay. Unlike previous work, OnehopMANET uses a P2P overlay that is capable of achieving lookups in a single hop. Through simulation we show that this approach offers performance benefits when compared with approaches which employ a multi-hop P2P overlay.
\end{abstract}

\section{INTRODUCTION}

P2P networking paradigms have gained popularity as they support different applications such as file sharing, voice over IP (VoIP), and messaging without the use of centralised servers. P2P networking systems are usually implemented as an overlay networks that allow higher-layer communication among participating peers. The established connections between peers in the overlay are usually underlayindependent. The original architecture of $\mathrm{P} 2 \mathrm{P}$ is mainly intended to operate in infrastructure (wired) networks. However, the rapid development in wireless communication technology has brought a need for the adoption of peer to peer network systems into the mobile field [1].

Mobile ad hoc networks (MANET) are a type of wireless network which when combined with $\mathrm{P} 2 \mathrm{P}$ overlays can benefit from the synergies [18]. MANETs are composed of a set of autonomous mobile nodes that communicate with each other using wireless connections without relying on any pre-existing infrastructure. In such networks, a node acts as a client and a server. In addition, the participating nodes collaborate with each other through forwarding messages towards other nodes.

There are many common characteristics between P2P overlay networks and mobile ad hoc network. Selforganization, decentralization, dynamicity and changing topology are key shared features. As a result of having similar characteristics, they face common challenges, most notably to maintain connectivity in dynamic and decentralized networks. However, the challenges are seen to be stronger when deploying P2P over MANET. This is largely due to the lack of the rich services provided in the IP routing infrastructure.

In this paper we combine a proactive MANET routing protocol, Optimized Link State Routing protocol, (OLSR) [13] with EpiChord [12], a Distributed Hash Table (DHT) based P2P overlay network which can achieve lookups in a single hop. This is a novel approach as previous work focused on pairing multi-hop overlays with MANET. The resulting system, OnehopMANET employs a cross-layer approach to exchange information between the MANET and P2P overlay achieving a consistent one-hop lookup performance at a reduced bandwidth cost. In doing so, for the first time, we evaluate the performance of EpiChord in MANET. We use a packet level simulator (Omnet++) together with an implementation of MANET protocols (MANET-INET) and P2P overlay model (Oversim).

The rest of this paper is structured as follow: Section II introduces the mismatching problem between virtual overlay and physical topology followed by a review of related work in section III. The proposed system is introduced in section IV and then evaluated in section V. Finally, the conclusion and future work is presented in section VI.

\section{MISMATCH BETWEEN VIRTUAL AND PHYSICAL TOPOLOGY}

One key issue when combining a physical underlay network such as MANET with a DHT based overlay network is that a single hop in the overlay translates to a path in the underlay. Consequently, a single overlay hop results in multiple hops in the underlay. Often peers which are neighbors in the overlay are separated by many hops in the physical network. When using multi-hop P2P overlays in such a setting, each overlay hop results in multiple hops in the underlay. Progressing through the overlay path to the destination may well mean contacting some underlay nodes repeatedly and passing underlay nodes which are very close to the final destination node. Consequently, multi-hop overlays are not well suited to such systems. Rather one-hop overlays, avoid such inefficient routing paths. OnehopMANET is proposed to reach the destination in a one logical hop.

To illustrate the problem, Figure 1 shows a network topology of a MANET. If for example node 27 with logical ID 2000100 (see Figure 2) requests a key that resides on node 11 with 
logical ID 2000600, it may go through a number of overlay hops (red path) until node 11 is reached. However, in the underlay, node 11 can be reached in a single physical hop since it is close by. Thus, one-hop overlays directly benefit from efficient underlay routing and avoid crisscrossing the MANET network to reach a peer that might be close in the physical network.

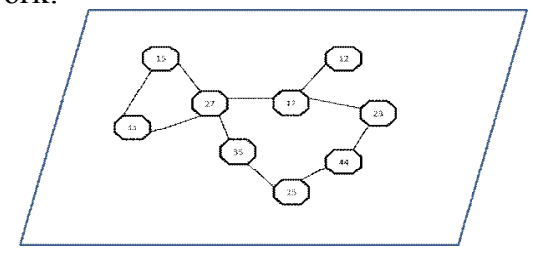

Figure 1: MANET Network.

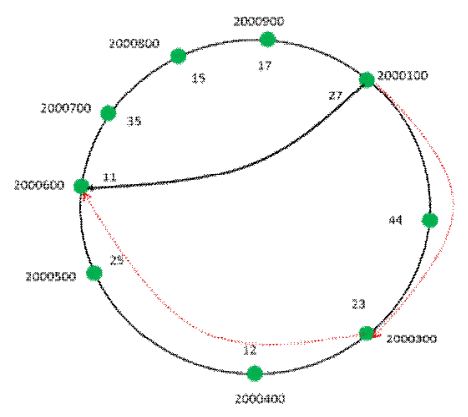

Figure 2: Logical Overlay over MANET Network.

\section{RELATED WORK}

Structured P2P overlays typically employ a Distributed Hash Table (DHT) based approach. A review and analysis of such systems can be found in [19], [20], and [21]. Most DHT based overlays require multiple hops, however, some systems such as D1HT and EpiChord [12] can achieve lookups in a single hop. The approach in this paper employs EpiChord, which is a DHT algorithm where peers maintain a full routing table and ideally approach $\mathrm{O}(1)$ hop lookup performance compared to the $\mathrm{O}(\log \mathrm{N})$ hop performance offered in many multi-hop networks. EpiChord is based on the Chord DHT and organized as a one-dimensional circular space where each node is assigned a unique node identifier. The node responsible for a key is the node whose identifier most closely follows the key. In addition to maintaining a list of the $\mathrm{k}$ succeeding nodes, EpiChord also maintains a list of the $\mathrm{k}$ preceding nodes and a cache of nodes. Nodes update their cache by observing lookup traffic. Therefore nodes add an entry anytime they learn of a node not already in the cache and remove entries which are considered dead.

MANET routing protocols can be divided into unicast, multicast and geocast approaches. For this paper, the unicast approaches are of interest. These can be divided into proactive, reactive and hybrid routing protocols [24].

Reactive routing protocols discover a path to the destination node as required. Once discovered, the route is maintained until no longer required or being unavailable. The disadvantage of reactive routing is the relatively long delay during route discovery. On the other hand, in proactive routing protocols each node holds routing information to all other nodes. OLSR Optimized Link State Routing protocol[13] is an example of proactive routing protocols. Proactive routing approaches exhibit lower routing latency than reactive systems, however they incur increased overhead traffic. When combining P2P overlays with MANET systems, the lower overhead of reactive systems is offset by the frequent route requests by overlay nodes. Thus the additional cost of proactive systems when combined with $\mathrm{P} 2 \mathrm{P}$ overlays is minimal. Hence the approach presented in this paper employs proactive MANET routing.

A number of systems combining P2P overlays with MANETs have been proposed. However, a number of these approaches have not considered mobility nor has their performance been evaluated either through simulation or testbed experimentation.

A number of approaches have extended or modified existing P2P systems to work on MANET. For instance, Scalable Source Routing SSR [2] builds a Chord-like ring at the network layer. SSR nodes maintain physical neighbours, virtual successors list, virtual predecessors list and cached information. Virtual Ring Routing VRR [3] organizes nodes into a virtual ring similar to Chord and Pastry at the network layer. It supports traditional point to point routing and DHT routing as well. MADPastry [4] integrates Pastry with the reactive MANET protocol AODV. It uses random landmarking, where a set of nodes in the same physical cluster share a common overlay ID, to consider local proximity.

CrossROAD [5] adopted Pastry over OLSR. Each CrossROAD node maintains a global services table that stores all the services provided in the network. Ekta [6] also integrates Pastry with DSR[7] at the network layer. It overhears the underlay control messages to reduce network traffic. Some proposed systems adopt building minimum spanning trees to recognize the physical topology[8][9]. MANETChordGNP [10] considers the physical locality through using GNP global network positioning system. It integrates modified Chord that uses GNP with AODV. Enhanced Backtracking Chord [11] modified Chord to perform better in MANET. It modified Chord to use retransmission and path selection.

\section{PROPOSED SYSTEM (ONEHOPMANET)}

Unlike previous approaches, OnehopMANET combines a onehop structured P2P overlay (EpiChord[12]) with a proactive MANET protocol (OLSR [13]). Figure 3 depicts the architecture of the proposed system.

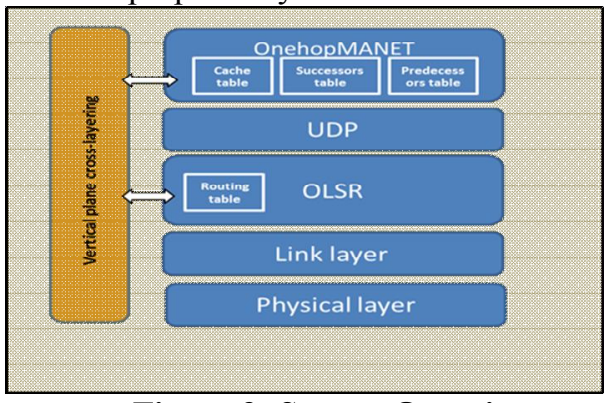

Figure 3: System Overview 
Each node in OnehopMANET assigns itself a unique identifier by hashing its own IP address. OnehopMANET builds a one dimensional circular address space as in EpiChord. A key is stored on the node that most closely follows the key. Similar to EpiChord, OnehopMANET maintains lists of key nodes that succeed and precede a node. In addition, it also maintains a cache table. Each node in OnehopMANET updates its cache table largely by using information from the proactive underlay. OnehopMANET inherits from EpiChord the O (log $\mathrm{N})$ performance, in the worst case, and also the potential of $\mathrm{O}(1)$ performance if up to date routing information is available. Due to the rich cache table, OnehopMANET achieves one hop performance (see Section V).

OnehopMANET uses cross-layering to exchange information between the underlay and overlay to reach one hop performance. This approach reduces the typical overhead from employing one hop overlay systems as routing updates from the underlay are forwarded to the overlay which in turn can scale down its own update mechanisms. OnehopMANET follows the manager method of cross-layering [14] to optimize the network layer's routing information transfer.

A notification board is used as a vertical plane that manages sharing the information between the application and the network layer. OLSR notifies the overlay whenever changes occur in any of its routing tables, and provides the overlay with the updated contents. The overlay then uses this information to update its view of the network. More specifically, each peer maintains a successor list, predecessor list and a cache table. The update of these tables is mainly driven by underlay routing updates forwarded to the overlay. After any changes to routing tables in the underlay, a notification will be sent to the overlay peer to allow it to take the change into account in its own cache and successor and predecessor lists.

Consequently, using the underlay information significantly reduces the need for overlay maintenance traffic. OnehopMANET nodes do not initiate any joining messages. Once a peer gets information through the cross layer channel, it calculates the logical IDs of other peers by hashing their IP addresses and then populating routing table and cache table.

Besides the updates from the underlay, OnehopMANET can also use EpiChord lookup queries to update its routing tables. Each lookup query response contains some information from the queried peer. The need to use additional lookup messages to update peers' routing tables is however, much reduced in OnehopMANET.

Like EpiChord, OnehopMANET uses an iterative lookup algorithm where the queried peer will respond with its best knowledge of the queried key without forwarding the lookup to other nodes. EpiChord supports parallel lookups to increase its chance of finding the key with the first hop reducing lookup latency. However, OnehopMANET does not make use of this technique and only sends lookup requests to single destinations reducing the bandwidth usage. This is possible due to highly accurate routing information held by the peers.

\section{PERFORMANCE EVALUATION}

To evaluate the performance of OnehopMANET, we implemented the system in a network simulator which includes a packet level simulator together with an implementation of the MANET protocol (OLSR) in combination with an overlay simulator model of EpiChord. We used the discrete event simulation system OMNet++[16], the communication network simulation package INET-MANET[17] together with Oversim for the P2P model.

\section{A. Simulation Setup}

Table 1 shows the used parameters for the simulated scenarios. All the simulated scenarios were repeated five times and the result is the average of the repetitions. The used mobility model is Random Way Point model which is commonly used for simulating ad hoc networks. According to the Random Way Point mobility model, when a node travels from one position to another, it pauses for a time which is known as the pause time. In these simulations, the pause time is a random number between 1 and 50 seconds. For each of the simulated scenario, a network is given about 120 second to stabilize. After the 120 second, the measurements of the metrics begin.

TABLE I. SIMULATION CONFIGURATIONS

\begin{tabular}{|l|l|}
\hline Simulator & OMNeT++ \\
\hline Underlay routing protocol & OLSR \\
\hline Topology size & $1000 \mathrm{~m} \times 1000 \mathrm{~m}$ \\
\hline Number of mobile nodes & $30,50,70,90$ \\
\hline Mobility model & Random way point \\
\hline Node speed & $5 \mathrm{~m} / \mathrm{s}$ \\
\hline Pause time & Random between 1 and $50 \mathrm{sec}$. \\
\hline Measurement time & 600 seconds \\
\hline Transmission range & $250 \mathrm{~m}$ \\
\hline Network stabilization time & $120 \mathrm{~seconds}$ \\
\hline Lookup interval & $30 \mathrm{~s}, 10 \mathrm{~s}, 5 \mathrm{~s}$ \\
\hline Parallelism & 3 for EpiChord only \\
\hline Simulation repetitions & 5 \\
\hline
\end{tabular}

B. Performance metrics

The following performance metrics are evaluated from the conducted simulations:

- Lookup Success Ratio: The percentage of successful lookups in the overlay.

- Mean Network Traffic Load: The mean number of packets sent in the network including both, the MANET layer management traffic and the overlay traffic.

- Hop Count: The mean number of overlay hops required to retrieve a stored key. 


\section{Experimental Results}

We compare the performance of OnehopMANET with basic layered systems of EpiChord over OLSR (no cross-layer communication) and Chord layered over OLSR (no crosslayer communication) in networks of 30, 50, 70 and 90 nodes. In all simulated scenarios nodes were moving with a speed of $5 \mathrm{~m} / \mathrm{s}$ within the topology size of $1000 \mathrm{~m} \times 1000 \mathrm{~m}$.

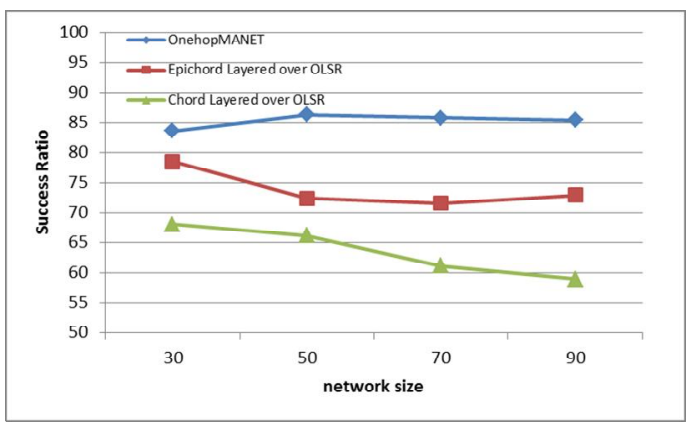

Figure 4 : Success ratio with lookup frequency of $30 \mathrm{~s}$.

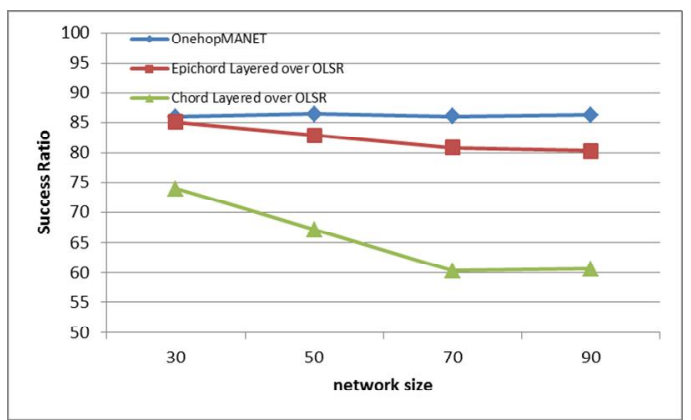

Figure 5: Success ratio with lookup frequency of $10 \mathrm{~s}$.

Figures 4, 5 and 6 show the success rate for the three systems with three different lookup intervals (Fig 4: 30s, Fig 5: 10s, Fig 6: 5s). OnehopMANET outperforms Chord and EpiChord in all the three different scenarios. As can be seen OnehopMANET maintains a $85 \%+$ performance across all network sizes and lookup frequencies. This means that unlike EpiChord, OnehopMANET is not dependent on additional lookup messages (and the routing table updates in the responses) to achieve a high degree of lookup success. The basic EpiChord/OLSR layered system achieves a comparable performance to OnehopMANET only with the highest lookup frequency of 5s. Clearly this comes at a cost of increased traffic in the network as can be seen in Figures 7,8 and 9.

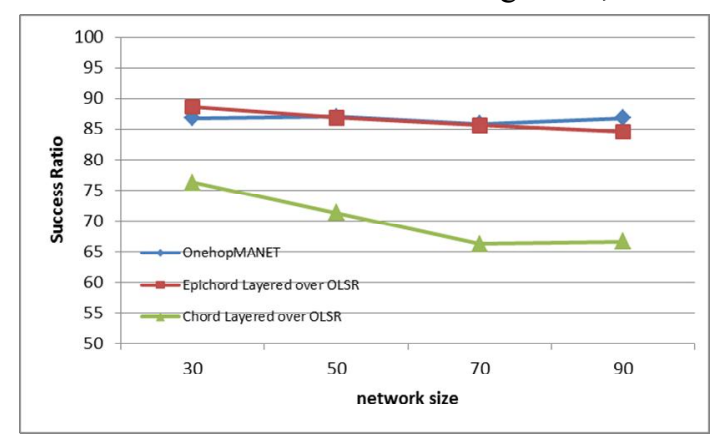

Figure 6 : Success ratio with lookup frequency of $5 \mathrm{~s}$.

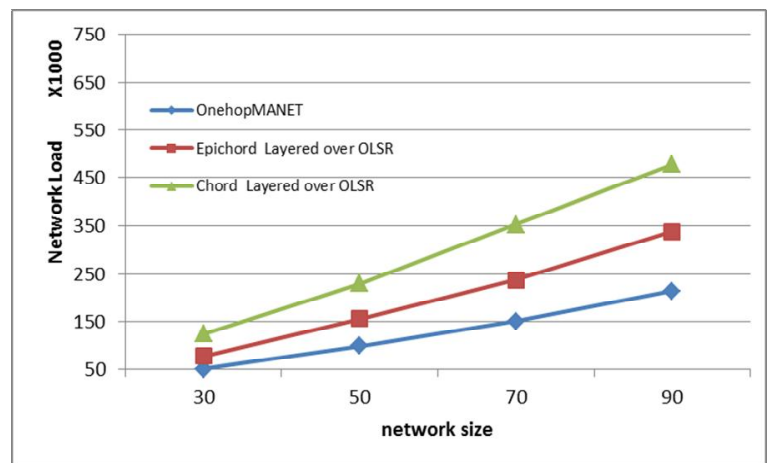

Figure 7: Network load with lookup frequency of 30s.

Figures 6-8 show the total traffic that was generated in the simulated networks with 30, 10 and 5 seconds lookup frequency. As the figures show, OnehopMANET causes the least amount of traffic in all scenarios (about half of the layered EpiChord/OLSR system).

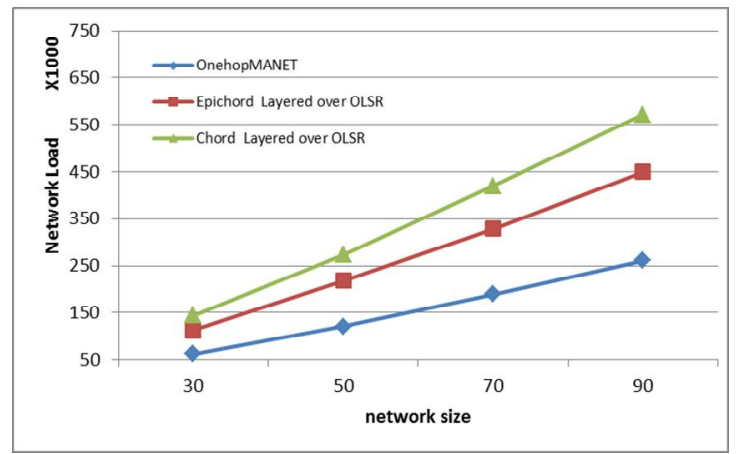

Figure 8: Network load with lookup frequency of $10 \mathrm{~s}$.

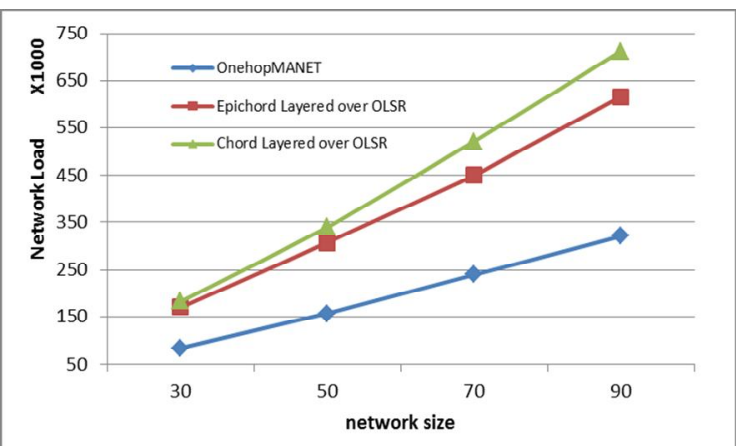

Figure 9: Network load with lookup frequency of 30s.

The final performance metric that was investigated is the overlay hop count. One of the aims of the proposed system is to achieve one logical hop for lookups avoiding inefficient lookup forwarding in the underlay. Figures 9, 10 and 11 show the logical hop count results for the three sets of simulated scenarios. OnehopMANET show a consistent hop count performance of lindependent of the network size and lookup frequency parameters. This indicates that OnehopMANET maintains highly accurate information in its routing tables. 


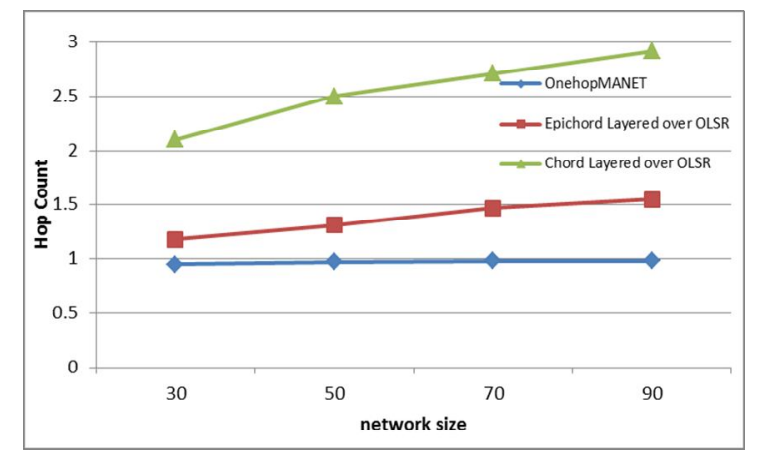

Figure 10: Logical path length with lookup frequency 30 s.

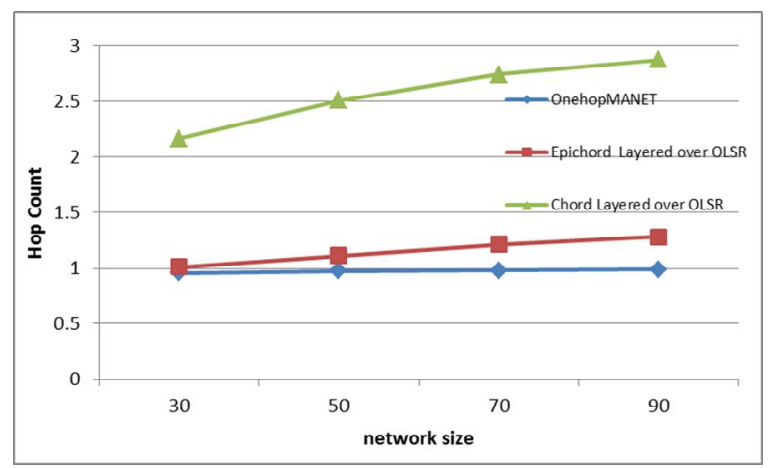

Figure 11: Logical path length with lookup frequency $10 \mathrm{~s}$.

Interestingly, the two other systems simulated show a declining performance as the network size increases. The layered EpiChord/OLSR system can improve its performance with an increased lookup frequency. But even at the highest lookup rate, this system declines as the network size increases. Despite its 3 parallel lookups, it cannot outperform OnehopMANET in any of the configurations.

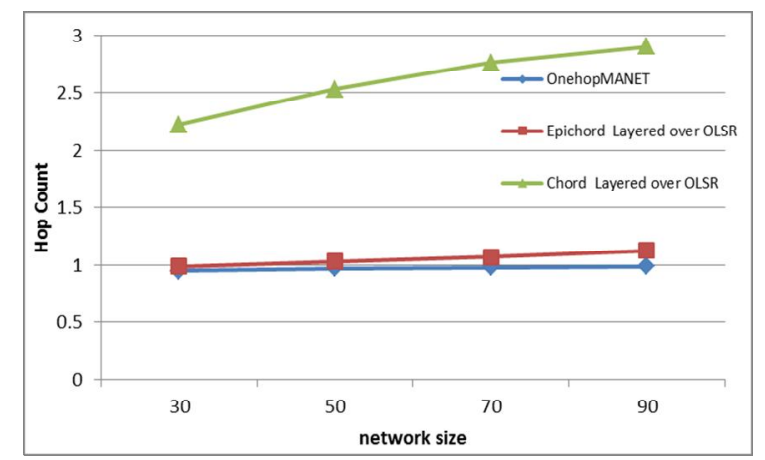

Figure 12: Logical path length with lookup frequency 5 s.

\section{CONCLUSION}

The most challenging problem when deploying P2P overlays over mobile ad hoc networks is managing the topology differences between the overlay and MANET underlay. In this paper, we have proposed a solution through the use of a onehop logical overlay that avoids inefficient routing in the underlay. OnehopMANET employs cross-layer communication exchanging routing information updates between the MANET and P2P layers exploiting the synergy between both systems. A performance evaluation was conducted using a simulation framework combining a packetlayer MANET model with a P2P overlay model. The simulation results show that OnehopMANET achieves a consistent one-hop performance across all simulated network sizes and lookup frequencies.

\section{REFERENCES}

[1] F. Chowdhury and M. Kolberg, "A Survey of Peer-to-Peer Solutions in Mobile and Cellular Networks," PGNet, 2012.

[2] T. Fuhrmann, P. Di, K. Kutzner, C. Cramer, Pushing chord into the underlay: Scalable routing for hybrid manets. 2006.

[3] M. Caesar, M. Castro, and E. Nightingale, "Virtual ring routing: network routing inspired by DHTs," ACM SIGCOMM 2006.

[4] T. Zahn and J. Schiller, "MADPastry: A DHT substrate for practicably sized MANETs," Proc. ASWN, 2005.

[5] F. Delmastro, "From Pastry to CrossROAD: CROSS-layer Ring Overlay for $\mathrm{AD}$ hoc networks," in $3^{\text {rd }}$ IEEE International Conference on Pervasive Computing and Communications Workshops, 2005.

[6] H. Pucha, S. Das, and Y. Hu, "Ekta: An efficient dht substrate for distributed applications in mobile ad hoc networks," $6^{\text {th }}$ IEEE workshop on mobile computing systems and applications (WMCSA), 2004.

[7] D. Johnson, D. Maltz, and J. Broch, "DSR: The dynamic source routing protocol for multi-hop wireless ad hoc networks," Ad hoc Netw., pp. 1$25,2001$.

[8] N. Shah, D. Qian, and R. Wang, "MANET adaptive structured P2P overlay," Peer-to-Peer Netw. Appl., vol. 5, no. 2, Nov. 2012.

[9] S. a. Abid, M. Othman, N. Shah, "3D P2P overlay over MANETs," Comput. Networks, vol. 64, pp. 89-111, 2014.

[10] S. G. Fantar and H. Youssef, "Locality-aware Chord over Mobile Ad Hoc Networks," 2009 Glob. Inf. Infrastruct. Symp., pp. 1-6, Jun. 2009.

[11] M. Thaalbi, N. Tabbane, T. Bejaoui, A. Meddahi, "Enhanced Backtracking Chord protocol for mobile Ad hoc networks," Int. Conf. Commun. Inf. Technol., pp. 191-195, Jun. 2012.

[12] B. Leong, B. Liskov, and E. D. Demaine, "EpiChord: parallelizing the chord lookup algorithm with reactive routing state management," Proceedings. 2004 12th IEEE Int. Conf. Networks, pp. 270-276, 2004.

[13] T. Clausen and P. Jacquet, "Optimized link state routing protocol (OLSR)," RFC:3626, IETF, 2003.

[14] B. Fu, Y. Xiao, H. Deng, and H. Zeng, "A Survey of Cross-Layer Designs in Wireless Networks," IEEE Commun. Surv. Tutorials, vol. 16 , no. 1, pp. 110-126, 2014.

[15] I. Baumgart, B. Heep, and S. Krause, "OverSim: A Flexible Overlay Network Simulation Framework,” IEEE Glob. Internet Symp., 2007.

[16] “OMNET++ network simulator." [Online]. Available: http://www.omnetpp.org/.

[17] "INET FRAMEWORK." [Online]. Available: http://inet.omnetpp.org/.

[18] M. Gerla, C. Lindemann, and A. Rowstron, “P2P MANET's-New Research Issues.,” Dagstuhl Seminar 05152, Dagstuhl Seminar Proceedings, ISSN 1862-4405, 2005.

[19] J. Li, J. Stribling, R. Morris, M. Kaashoek, T. Gil, “A performance vs. cost framework for evaluating DHT design tradeoffs under churn", 24th IEEE Infocom, vol. 1, 2005, pp. 225-236.

[20] I. Baumgart and B. Heep. "Fast but economical: A simulative comparison of structured peer-to-peer systems", 8th Euro-NF Conference on Next Generation Internet. IEEE, June 2012.

[21] F. Chowdhury, M. Kolberg. "Performance Evaluation of Structured Peer-to-Peer Overlays for Use on Mobile Networks", 6th IEEE Conference on Developments in eSystems Engineering (DESE), 2013.

[22] F. Chowdhury, M. Kolberg. Performance Evaluation of EpiChord under High Churn, 8th ACM workshop on Performance Monitoring and Measurement of Heterogeneous Wireless and Wired Networks ,2013.

[23] J. Furness, F. Chowdhury, M. Kolberg. An Evaluation of EpiChord in OverSim, 5th Intl. Conf. on Networks \& Communications, Lecture Notes in Electrical Engineering, Meghanathan, N.; Nagamalai, D.; Rajasekaran, S. (Eds.), Springer, Vol. 284, 2014.

[24] R. R. Roy, Handbook of mobile ad hoc networks for mobility models. Boston, MA: Springer US, 2011, pp. 3-22. 